\title{
Diabetes y Embarazo
}

\section{Consideraciones Generales y Manejo Revisión Bibliográfica}

\author{
Dr. Jorge Elías Peláez Vallejo
}

Universidad de Antioquia, Hospital Universitario San Vicente de Paúl, Facultad de Medicina.

\section{INTRODUCCION}

El manejo de la diabética embarazada ha sufrido modificaciones a través del tiempo debido al mejor conocimiento de su fisiopatología. A pesar de una incidencia de sólo $1.02 \%$ (Raymond Corwin), las complicaciones que esta patología ocasiona al binomio madre - feto son importantes, hecho que ha llevado a las diferentes escuelas a profundizar sobre el tema con el fin de realizar un adecuado manejo tendiente a disminuirlas, objetivo que se ha logrado especialmente en la mortalidad perinatal y un poco menos en la morbilidad.

La mortalidad perinatal, que según diferentes autores puede variar entre el 20 y el $30 \%$, se ha logrado disminuir a un $5 \%$. Adaski y colaboradores lograron disminuir su mortalidad de un $2.8 \%$ al $0 \%$ en 18 meses. Corwin en una revisión de 10 años relata una mortalidad del $12.4{ }^{\circ}$ o. Pederson en un estudio de 220 embarazadas diabéticas informa tasas de mortalidad perinatal del $4.6 \%$. La pro-
Departamento de Ginecología y Obstetricia Medellín, 1981.

porción de muertes perinatales está en franca relación con el grado de diabetes y con los factores agravantes de Pederson, y es claro cómo, un mal control metabólico durante la gestación, conlleva mayores tasas de mortalidad.

Las malformaciones congénitas, la macrosomía fetal, el síndrome de dificultad respiratoria, la hipoglicemia y la hipocalcemia, son los mayores problemas en los hijos de madres diabéticas; se ha demostrado cómo el manejo dietético solo no es suficiente para bajar esta morbilidad, por lo que se hace necesario el uso de la insulina, aspecto éste de importancia en lo referente a las malformaciones congénitas, donde se ha logrado una incidencia de un $4.6 \%$ con un manejo adecuado dietético e insulínico.

Caso similar sucede con la macrosomía fetal que es muy frecuente cuando las concentraciones de glucosa en sangre están elevadas. Las malformaciones congémitas tienen un agravante mayor y es el relacionado con la dificultad de un tra- 
tamiento oportuno, pues la mayoría se producen en las primeras semanas de gestación, cuando no se ha diagnosticado la enfermedad, o si ya se conoce, no se sabe exactamente el momento de la fecundación. Lo anterior retarda los controles prenatales y no permite evitar esta complicación; se considera de importancia el uso de la $\mathrm{Hb}$ A1c como medio diagnóstico para conocer el control de la diabética en los primeros días de gestación y descartar las posibilidades de malformaciones congénitas. Sabemos que la $\mathrm{Hb}$ A1c sirve como indicador de control de enfermedad en los últimos 40 días a la toma de su muestra.

El síndrome de dificultad respiratoria tiene una incidencia elevada $\mathrm{y}$ puede alcanzar hasta un $28 \%$; es mayor en hombres que en mujeres. Esta cifra podría rebajarse con un buen control de la madre diabética.

La hipoglicemia suele presentarse en las 3 primeras horas del nacimiento, es asintomática y menos del $10 \%$ de recién nacidos presentan signos y síntomas inespecíficos. Se considera hipoglicemia en los recién nacidos de término la cifra por debajo de $30 \mathrm{mgs} \%$ y menor de 20 mgs $\%$ para los de bajo peso.

Un $20 \%$ de los recién nacidos de madres diabéticas presentan hipocalcemia que suele ser transitoria; se desconoce la patogenia de esta alteración pero existe una correlación entre los niveles séricos de calcio con la edad gestacional y la clasificación de Priscilla White, limitada a las clases B a F. Se considera hipocalcemia la existencia de valores por debajo de $3.5 \mathrm{mEq} / \mathrm{ml}$.

No sólo macrosómicos sino también niños de bajo peso podemos encontrar como productos de madres diabéticas, en especial en aquellas embarazadas con diabetes vascular, explicándose esta alteración por una lesión a nivel placentario con disminución del espacio intervelloso.
La mortalidad materna no es alta y puede oscilar alrededor del $0.5 \%$ (Pederson y colaboradores). Los siguientes trastornos se pueden relacionar con las complicaciones o la morbilidad materna que conllevan a una alta tasa de morbimortalidad perinatal.

1. Complicaciones vasculares: son graves; la retinopatía progresa durante el embarazo. Con tratamiento puede haber remisión en el postparto. Otros, como Fukuda (1979), consideran que el embarazo per se no agrava la retinopatía diabética.

2. Complicaciones renales: La incidencia de infecciones urinarias oscila entre el $16 \mathrm{y}$ el $21.7 \%$, incluyendo la pielonefritis (Pederson). La bacteriuria asintomática determinada precozmente en la embarazada diabética, se asocia a pielonefritis clínica, parto prematuro y muerte fetal; esta bacteriuria se correlaciona con las clases D a F de Priscilla White.

3. Gestosis: La incidencia de eclampsia en diabética embarazada fue estimada en un $2 \%$. La toxemia se considera signo de mal pronóstico. (Arismendi Tavares).

4. Hidramnios: Su frecuencia es 10 veces mayor en la embarazada diabética, se explica por un mayor aumento de la concentración de glucosa en el líquido amniótico.

5. Otras pueden estar relacionadas con el parto prematuro y la ruptura prematura de las membranas.

La enfermedad tiroidea, si bien no es una complicación de la diabética embarazada no debe pasar desapercibida, pues en un estudio de Norman G. Soler y col. encontraron una incidencia de $5 \%$ de diabetes en mujeres con antecedente de enfermedad tiroidea. Refieren que estas pacientes pueden presentar esteri- 
lidad susceptible de tratamiento exitoso y durante el embarazo desarrollar una diabetes.

CLASIFICACION: Diversas clasificaciones se han publicado para enfocar el estudio de la gestante diabética. Sin embargo, la de Priscilla White, combinada con los factores agravantes de Pederson, es la más aceptada.

La Organización Mundial de la Salud, reunió en Génova una serie de expertos en diabetes en el año de 1980 para tratar el tema en mención. La clasificación recomendada fue la siguiente:

\section{A. Clases Clínicas}

Diabetes Mellitus

Tipo I; Insulino - dependiente

Tipo II: No insulino - dependiente

\section{a) No obesa \\ b) Obesa}

Otros tipos: incluye diabetes mellitus asociada con ciertas condiciones y síndromes: Pancreatitis, enfermedad hormonal, drogas o condiciones químicas inducidas, anormalidad de los receptores insulínicos, ciertos síndromes genéticos $y$ otras.

a) No obesa

b) Obesa

c) Intolerancia a la glucosa asociada con ciertas condiciones y síndromes.

Diabetes gestacional

\section{B. Con riesgo estadístico}

Anormalidad previa. de curva de tolerancia a la glucosa

Cuadro No. 1

\section{CLASIFICACION WHITE}

\begin{tabular}{|c|c|c|c|c|c|c|}
\hline A & - & \multicolumn{2}{|c|}{$\begin{array}{l}\text { Curva tolerancia a la } \\
\text { glucosa anormal }\end{array}$} & $\mathrm{F}$ & - & Nefropatía clínica \\
\hline B & - & $\begin{array}{r}\text { Comienzo } \\
>20 \text { años } \\
\end{array}$ & $\begin{array}{l}\text { Duración } \\
<10 \text { años }\end{array}$ & $\mathrm{R}$ & - & $\begin{array}{l}\text { Retinopatía proli- } \\
\text { ferativa }\end{array}$ \\
\hline $\mathrm{C}$ & - & $\begin{array}{c}10-20 \\
\text { años }\end{array}$ & $\begin{array}{l}10-20 \\
\text { años }\end{array}$ & $\mathrm{G}$ & - & $\begin{array}{l}\text { Múltiples embara- } \\
\text { zos pérdidos }\end{array}$ \\
\hline D & - & $<10$ años & $>10$ años & $H$ & - & $\begin{array}{l}\text { Arterioesclerosis } \\
\text { coronaria }\end{array}$ \\
\hline$E$ & - & \multicolumn{2}{|c|}{$\begin{array}{l}\text { Calcificaciones } \\
\text { pélvicas }\end{array}$} & 1 & - & $\begin{array}{l}\text { Embarazo con tras- } \\
\text { plante renal }\end{array}$ \\
\hline
\end{tabular}

\section{Cuadro No. 2}

\section{FACTORES AGRAVANTES DE PERDERSON}

1) Pielonefritis crónica

2) Toxemia

3) Precoma: Acidosis clínica y severa

4) Negligencia: Psicópata, captación antes de 60 días de la fecha probable del parto
Anormalidad potencial de tolerancia a la glucosa

Dentro de esta clasificación se deben anexar las clases de diabetes de Priscilla White y los factores agravantes de $\mathrm{Pe}$ derson, con el fin de tener una exacta ubicación de la diabética embarazada. 
En los cuadros No. 1 y 2 se presentan cada una de estas.

\section{Manejo de la Diabética Embarazada}

El manejo adecuado de una patología requiere, entre otras cosas, la detección precoz de ella y en el caso de la gestante diabética existen factores que nos ayudan a sospecharla y detectarla con base en un interrogatorio para conocer la existencia de:

1. Antecedentes hereditarios o historia familiar

2. Antecedentes de macrosomía fetal

3. Historia de enfermedad tiroidea $y$ toma de anovulatorios

4. Antecedente de hidramnios, ruptura prematura de membranas o parto prematuro.

5. Niños de bajo peso (Diabetes vascular)

6. Antecedente de toxemia

7. Diferencia entre crecimiento uterino y fecha de amenorrea

8. Antecedente de mortinatos

9. Mujer gestante de más de 25 años

10. Antecedente de glicemias con alguna alteración

11. Obesidad, hipertensión, coronariopatía

12. Hijos con malformaciones congénitas

13. Antecedente de hijos con síndrome de dificultad respiratoria

14. Infecciones a repetición (Pielonefritis Micosis).

La sospecha de enfermedad por la existencia de uno o varios de los factores enunciados nos obliga a confirmar el diagnóstico. Lo anterior se puede hacer con una curva de tolerancia a la glucosa; una sola glicemia o glicemia pre y postprandial no son suficientes para descartar la enfermedad.

La curva de tolerancia a la glucosa debe realizarse después de 2 días o más de una dieta libre que incluya más de 300 gramos/día de hidratos de carbono. El día de la prueba se suministran a la paciente 100 gramos de glucosa oral, en 500 cc de agua con el jugo de 1 limón; se toman muestras de sangre venosa en ayunas a los $30-60$ y 120 minutos después de la ingestión, siendo los valores respectivos máximos:

Cuadro No. 3

\begin{tabular}{|ccc|}
\hline & SANGRE VENOSA & PLASMA \\
EN MGRS & EN MGRS \\
En ayunas & 90 & 110 \\
$30^{\prime}$ & 165 & 170 \\
$60^{\prime}$ & 145 & 170 \\
$120^{\prime}$ & 125 & 145 \\
\hline
\end{tabular}

Se considera prueba normal cuando todos los valores son menores de estas cifras; anormal cuando exista un sólo valor mayor de los enunciados y diabética cuando se encuentren dos o más valores elevados.

De estos resultados tendremos la embarazada con curva normal pero con factores de riesgo y la diabética A ogestacional, diagnóstico este último que sólo se podrá confirmar en el puerperio, ya que esta paciente puede ser una diabética que se diagnóstico con la gestación o una simple diabetes gestacional que desapareció luego de su embarazo. 
Para la embarazada con diabetes diag. nosticada previamente, se debe hacer en el momento de la primera consulta prenatal una prueba de tolerancia oral, tomando una muestra en ayunas y otra a las dos horas; deben obtenerse valores máximos en sangre venosa de 120 mgrs en ayunas y 180 mgrs a las dos horas; valores más altos nos indican que la paciente está descompensada; a estas pacientes se les pide además examen de orina para conocer la existencia de cuerpos cetónicos.

Donde haya posibilidad de realizar el estudio de $\mathrm{Hb} \mathrm{A1c}$, se debe solicitar a toda mujer embarazada con diabetes previamente diagnosticada.

Confirmado el diagnóstico, debe planearse el mariejo adecuado de la paciente con un esquema que incluya: frecuencia de consultas prenatales con acciones específicas, dieta, droga y educación, esquema éste que depende del tipo de diabetes.

La frecuencia de la consulta prenatal debe hacerse quincenal hasta la semana 30 de gestación y luego cada 8 días, hasta el día en que se decida la terminación del embarazo; en estas consultas debe llevarse un control estricto del peso, solicitar glicemias en cada consulta y evaluar la dosis de insulina.

Es recomendable la hospitalización de la paciente en el momento de confirmación del diagnóstico, con el fin de determinarle la dieta y dosis de insulina requeridas; esta hospitalización debe ser corta y se repetirá cada vez que la paciente presente el más mínimo problema; es recomendable una hospitalización en cada trimestre. La educación es un factor esencial para que la paciente conozca su enfermedad y ayude al manejo adecuado de su patología; en el aspecto educativo debe hacerse énfasis en la dieta y la importancia de practicarse -los examenes requeridos.

La consulta prenatal igualmente debe estar destinada a prevenir y diagnosticar patología dependiente $o$ independiente de la gestación: hipertensión, coronariopatía, insuficiencia renal e infecciones.

En relación con la dieta, ésta debe hacerse en forma individual para cada paciente, teniendo en cuenta sus hábitos alimenticios y el nivel social económico. La base del tratamiento es llevar los niveles glicémicos de la gestante diabética como si no fuese diabética. A las obesas no se les debe hacer perder mucho peso y las delgadas no deben ganar más peso que el acostumbrado en el embarazo normal. Por término general, la dieta debe calcularse con base en 30 a 35 calorías por kilogramo por día $(1.800$ a 2.200 calorías día) repartido en $24 \%$ al desayuno, $30 \%$ al almuerzo, $33 \%$ a la comida y $13 \%$ al acostarse.

El $45 \%$ del valor calórico total será suministrado como hidratos de carbono. La ingesta de proteínas debe ser de 1.5 gramos por kilo de peso y el resto aportado en forma de lípidos.

La insulina debe aplicarse (la N P H) en dosis de $20 \mathrm{U}$. repartida las $2 / 3$ partes en la mañana y la tercera parte en la tarde; la insulina cristalina en $10 \mathrm{U}$. en la mañana; esta dósis debe adecuarse a la respuesta de cada paciente cuando está hospitalizada, con controles de glicemia cada 6 horas; una vez la paciente ambulatoria se seguirá su control con glicemias cada 8 días y destrostix diario luego de cada comida, con instrucciones claras de vigilar cualquier cambio comunicando inmediatamente a su médico.

Los hipoglicemiantes orales deben suspenderse por completo por los efectos colaterales que traen para el feto, como lo es la hipoglicemia y las malforma- 
ciones congénitas. Además, dificultan el control de las glicemias.

Algunos autores consideran que la piridoxina hace parte del tratamientosobre la base de una mejor tolerancia a los carbohidratos, debido a la excreción excesiva de ácido xanturénico; en este punto no se encuentra consenso general de los resultados obtenidos (32).

METODOS DE VALORACION DE BIENESTAR FETAL: Si bien el manejo de la gestante diabética ya ha sido analizado, existe otro aspecto de igual importancia como es el conocer el estado fetal a través de la gestación; esta es la razón por la cual se recomiendan diferentes exámenes especiales:

1. Ecografía: Debe practicarse un primer estudio antes de la semana 20 de gestación, para detectar malformaciones congénitas, confirmar edad gestacional y tomar perímetro craneano y abdominal, con el fin de tener un punto de referencia para evaluar el crecimiento fetal. A la semana 28 se hará un segundo examen $y$ de la semana 32 en adelante, estudios cada 2 semanas con el fin de evaluar crecimiento fetal. Fescina mostró cómo la ecografía a través del perímetro abdominal detectaba a tiempo el niño macrosómico y los pequeños para la edad gestacional. En los niños macrosómicos la ecografía nos permite observar un doble contorno alrededor del tronco y la cabeza por separación de las capas del tejido, debido a depósitos de grasa o a edema subcutáneo; este es un signo de mal pronóstico que obliga a terminar la gestación si existe madurez pulmonar.

2. Prueba sin estímulo: Prueba de gran importancia en el manejo de la embarazada de riesgo, se recomienda en la gestante diabética a partir de la semana 32 y repetirla cada 8 días en caso de ser reactiva; la prueba no reactiva debe repetirse a las 12 horas; de persistir el resultado debe recurrirse a la prueba de la oxitocina, que de ser positiva, se seguirá con una prueba de Pose que nos indicaría la terminación del embarazo.

3. Estriol: Se recomienda la dosificación del estriol sanguíneo por radio inmunoensayo dos veces por semana a partir de la semana 32, (Giussi) siendo el nivel de estriol de $56 \mathrm{ngr} / \mathrm{ml}$. Otros autores recomiendan mediciones periódicas del estriol urinario a partir de la semana 34 de gestación y cuando se encuentre una baja del $40 \%$, someter la madre a la prueba sin estímulo; si es reactiva continuar dosificando el estriol.

\section{Pruebas de madurez pulmonar:}

a) La prueba de la espuma o Clements no es el más indicado en la gestante diabética por su alta incidencia de dudosos y falsos negativos en comparación con pacientes con otra patología; sirve en el caso de ser positivo.

b) Relación - Lecitina - Esfingomielina: aceptada como un buen índice de madurez pulmonar cuando es mayor de dos, en la gestante diabética no lo es, pues se ha encontrado hasta un $28 \%$ de niños con inmadurez pulmonar cuando la relación fue mayor de dos. Se ha atribuido esta situación a una acelerada relación de L/E y no del fosfatidilglicerol que es uno de los fosfolípidos que ayudan a la estabilidad pulmonar.

En estudios comparativos con grupos control y madres diabéticas, se observó que entre la semana 34 y 38 la relación Fosfotidilglicerol/Fosfotidilinositol es más lenta en la diabética que en la embarazada normal y lo contrario sucede con la L/E. Es también conocido que la lecitina en el surfactante pulmonar contiene fosfatidilglicerol y fosfotidilinositol.

Gluck y colaboradores consideran que que los fetos de madres diabéticas tienen una acelerada maduración de L/E y no 
la PG/PI. Se considera también que una alteración de otras hormonas como la baja de catecolaminas, el glucagón, glucocorticoides anormales, pueden coexistir en los niños de madres diabéticas, por lo que es importante esclarecer el papel de otras hormonas en la maduración pulmonar.

De lo dicho anteriormente podríamos deducir que la relación L/E no es indicador de madurez pulmonar en la gestante diabética y se requiere de la relación PG/PI; sin embargo, este examen es difícil de practicar y fue así como (Bustos) extrapolando la presencia de fosfotadilglicerol sin necesidad de identificarlo en virtud de la correlación existente entre $L / E$ y aparición de $F G$ en líquido amniótico, pudo determinar que cuando la relación L/E es mayor de 3 el $90 \%$ posee Fosfotidil-Glicerol.

En resumen diremos que un Clements positivo, una relación L/E mayor de 3 o la presencia de $\mathrm{PG}$ nos indican que existe madurez pulmonar.

VIAS PARA TERMINAR EL EMBARAZO: El momento y la forma de terminar la gestación depende del tipo de diabetes y las complicaciones que se presenten en cada embarazada. En términos generales, se recomienda que la diabetes clase $A$, puede dejarse evolucionar hasta la semana 40 si existe un buen control.

Las clases B - C, hasta la semana 38 y las otras entre la semana 35 a 37 ; como requisito de terminación es necesario que exista madurez pulmonar, exceptuándose cuando exista peligro para la vida de la madre.

\section{Bibliografía}

1. MURATA K. GLUCOSE metabolism during and after pregnancy in normal
El parto se hará por vía vaginal cuando exista un cuello favorable para inducción y haya iniciado el trabajo de parto en forma espontánea, requiriéndose en ambos casos del monitoreo estricto durante el trabajo de parto.

Cuando no se presenten estas circunstancias se recomienda la cesárea. Raymond Corwin en un estudio retrospectivo de 10 años, encontró una incidencia de cesáreas del $69 \%$, incrementándose a un $79 \%$ cuando se excluía la clase $A$. En comparación con un $13.4 \%$ de la población general.

A las pacientes que se inicia inducción o se encuentran en trabajo de parto, se les aplica suero glucosado al $5 \%$ a 50 gotas por minuto y se tomarán glicemias cada 4 horas con el fin de determinar la necesidad de insulina.

La paciente que va a ser sometida a cesárea se le aplicará la insulina en la noche si la intervención es en las primeras horas de la mañana, de no ser así, se le aplica la tercera parte de la dósis de insulina en la mañana; al quirófano se llevará con solución salina y cuando el feto ha sido extraido puede iniciarse soluciones glucosadas.

En el post-operatorio o post-parto inmediato hacer controles de glicemia periódicamente cada 2 a 4 horas por la posibilidad de crisis hipoglicémicas; durante las primeras 12 a 24 horas se mantendrá con soluciones intravenosas y control clínico estricto.

La lactancia no está contraindicada en la madre diabética, por lo que debe iniciarla lo más pronto posible. 
2. JACKSON W.P.V. and COETZEE E,J. Glicosuria as on indication for glucose tolerance testing during pregnancy. S. AFR Med J. 1979 56/22.

3. SOSEKO I.R. et al. The infant of the diabetic mother. Correlation of increased cord C-peptide levels whit macrosomia and hipoglycemia. N. Engl. J. Med. $1979301 / 16$.

4. COETZEE E.J., JACKSON W.P.V. Diabetes newly diagnosed during pregnancy. A 4 year study at Groote Shuur Hospital. S. Afric Med. J. 1979 56/12.

5. LEVENO K.J. et al. Appraisal of "rigid" blood glucose control during pregnancy in the overtly diabetic women Am. J. Obstetric Gynecol 1979 135/7.

6. LENZ S. et al. The effet of ritodrine in carbohydrate and lipid metabolism in normal and diabetic pregnant women. Acta Endocrinol. (copenhagen) 1979 $92 / 4$.

7. GRUDZINSKAS J.G. et al. Circulating levels of pregnancy specific B1 glycoprotein in pregnancies complicated by diabetes mellitus. Br. J. Obstet Gynaecol $197986 / 2$.

8. CRUIKSHANK D.P. et al. Altered maternal calcium homeostasis in diabetic pregnancy. J. Clin. Endocrinol. Metab. 1980 60/2.

9. JERVELL J. et al. Outcome of pregnancies in diabetic mothers inc Norway 1967-76. Diabetologia 1980 L8./2.

10. PSCHERA $\mathrm{H}$. et al. Total cortisol and L/S. ratio in ammiotic fluid fluid in late pregnancies complicated by diabetes mellitus. S.W.E. Horm Metab. Res. 1979 $11 / 11$.

11. ANDREWS A.G. et al. Ammiotic fulid palmitic acid/stearaic acid ratios, Lecithin / Sphingomyelin ratios and palmitic acid concentrations in the assesment of fetal lung maturity in diabetic pregnancies Br. J. Obstet Gynaecol. 1979 86/2.

12. WHITTLE M.J. et al. Estriol in pregnancy. $\mathrm{VI}$ experience whith unconjugated plasma estriol assays and anteprtum fetal heart rate testing in diabetic pregnancies. Am. J. Obstet-Gynecol. 1979 $135 / 6$

13. HALMAN $M$. et al. Ammiotic fluid phospholipid as a predicator of fetal maturity in diabetic pregnancies. Obstetric Gynecolog 1979 54/6.

14. LOWENSOHN R.I. and GABBE S.G The value of Lecithin/ Sphingomyelin ratios in diabetes: A critical review. Am. J. Obstet-Gynecol. 1979 134/6.

15. ROTCHILD S.B. et al. Estriol determinations in diabetic pregnancies complicated by nephropathy. Am. J. Obstetric Gynecol. 1979 134/7.

16. COETZEEN E.J. and JACKSON W.P.V. Metformin in management of pregnant insulin - independent diabetes. S.A.F. Diabetologia 1979 16/4.

17. KURJAK A. et al. Ultrasonic assesment of fetal renal function in diabetic preg. nancy Diabetologia Croat. 1979 8/1.

18. LANGE J. et al. Clinical experience obtained from attention to and delivery of pregnant women whith diabetes mellitus with insulin treatment requiered. Zentralb Gynakol 1979 101/14.

19. SOLER N.G. et al. Diabetes and thyroid disease during pregnancy. ObstetricGynecolog. 1979 54/3.

20. BOSEG G. Reflections on the survillance of the pregnancie of diabetic. Rev. Fr. Gynecol. Obstetric. 1979 74/6.

21. NAGY G. et al. Lecithin - Sphingomyelin ratio its prognostic importance to 
pregnancy of diabetics. Zentralbl Gynakol. $1979101 / 14$.

22. PETERS F.D. et al. Treatment of diabetics during pregnancys whith DesPhe insulin: Preliminary results Dtsch. Med. Woghenschr 1979 104/27.

23. ROBINSON R.E. Clinical aspects of diabetes in pregnancy. Pstgrad. Med. J. 1979 55/643.

24. FRANZ M. Nutritional management in diabetic and pregnancy. Biadetes Care $19781 / 4$.

25. STEEL J.M. et al. Obstetric history of diabetics: its relevance to the aetiology of diabetes. Br. Med. J. 1/6174 1979.

26. SOLER N.G. et al. Diabetic pregnancy; management of diabetes on the day of delivery. Biabetologia 1978 15/6.

27. MARTIN T.R. et al. Over doabetes in pregnancy. Obstetric Gynecolog 1979 $133 / 3$

28. SOLER N.G. Neonatal morbidity among infants of diabetic mothers. Diabetes Care 1979 1/6.

29. CABRERO L. et. al Palmitic acid and $\mathrm{P} / \mathrm{S}$ index in insulin dependent diabetes. Clin. Invest. Ginecolog. Obstetric 1979 6/1.

30. WEISS PAM et al. The management of the fetus in diabetics by means of ammiotic fluid insulin. Klin Wochenschr. $197991 / 9$.

31. MILLS J.L. et al. Malformations inc infants of diabetics mothers occur before the seventh festational week. Implications for treatment. Diabetes 1979 28/4

32. GILLMER G. et al. Pyridoxine treatment of chemical diabetes in pregnancies. Am. J. Obstetric-Gynecolog. 1979 133/5.
33. SINGH Mn. et al. Comparasion of oral and intravenous glucose tolerance tests in the diagnosis of diabetes in pregnancy. Br. J. Obstett Gynaecol. 1978 83/7.

34 CUNNINGHAM et al. Ammiotic fluid Phosphotidylglycerol in diabetic pregnan. cy. Am. J. Obstetric-Gynecol. 1978 $131 / 7$.

35. PEDERSON J.F. et al. Early growth retardation in diabetic pregnancy. $\mathrm{Br}$. Med. J. 1979 1/6155.

36. NATTRASS M. A. glucose-controlled insulin infusion system for diabetic women during labour. Br. Med. J. 1978 2/6137.

37. DISTLER W. et al. Estriol reaction test for evaluation of the fetoplacental function: Preliminary dates on normal and diabetic pregnancies in the third trimester. Eur. J. Obstetric-Gynecolog 1978 $8 / 5$.

38. BRAZY J.E. et al. Ammiotic fluid cortisol in normal and diabetic pregnant women and its relation to respiratory disease in the neonate. Am. J. ObstetricGynecolog 1978 51/4.

39. WEISS PAM. Insulin levels in ammiotic fluid. Management of pregnancy in diabetes. Obstetric-Gynecolog. 1978 51/4.

40 YEAST J.D, et al. The use of contonuous insulin infusion for the peripartum management of pregnant diabetic women. Am. J. Obstetric-Gynecolog. 1978 31/8.

41. GOLDKRAND J.W. Unconjugated estriol and cortisol in maternal and cord serum and ammiotic fluid in normal and abnormal pregnancy. Obstetric-Gynecol. 1978 $52 / 3$.

42. CASSAR J. et al. Simplified management of pregnancy complicated buy diabetes. Br. J. Obstetric-Gynaekol. 1978 85/18. 
43. MUELLER-HEUBACH E. et al. Lecithin/Sphingomyelin ratio in ammiotic fluid and its value for the prediction of neonatal respiratory distress syndrome in pregnant diabetic women. Am. J. Obstetric-Gynecolog. 1978 130/1.

44. KLOOSTERMAN G. J. Diabetes and Pregnancy. Geneesk Beig. 1977. 39/4.

45. TCHOBROUTSKY C. et al. The lecithin/ Sphingomyelin ratio in 132 insulindependent diabetic pregnancies Am. J. Obstetric-Gynecolog. 1978 130/7

46. GABBE S.G. et al. Management and outcome of pregnancy in diabetes mellitus clases B to R. Am. J. ObstetricGynecolog 1977 129/7.

47. DRORY M I. Pregnancy in the clinical diabetic. A personal experience of 739 cases. Ir Med. J. 148 (2): 75-9, 1979 En: IM. May. 80 p. 579.

48. MOSTYN R.H. et al. Maturity-onset diabetes (Letter). Br. Med J. 2 (6 189) 550, sep. $1 / 79$.

49. ROVERSI, G.D. et al. A new approach to the treatenent of diabetic pregnan women. Report of 479 cases seen from 1963 to 1975. J. Cell Biol. 135 (5) 567-76, Nov. $1 / 79$.

50. ELDREDGE D L. Management of the pregnant diabetic 1979. J. Maine Med. Assoc 70 (II Suppl) 22-5, Nov. 79. En: IM Junio 80. p. 687.

51. HAUKKAMAA M. et al. Screening management and outcome of pregnancy in diabetic mothers. Obstet Gynecol. 55 (5) 596-602, May. 80.

52. CORWIN R S. Pregnancy complicated by diabetes mellitus in private practice. A review of ten years. Trans Pac Coast Obstet Gynecol Soc 46: 55-60, 1979 En: IM. Aug. 80 p. 645.
53. MC FARLAND $K F$. Care of the pregnant diabetic. Postgradu Med 66 (5): 64-8, 70-1 Nov. 79 .

54. SANTIAGO J. J. et al. Studies with a pancreatic beta cell simulator in thethird trimester of pregnancis complicated by diabetes. Am J Obstet Gynecol. 132 (4): 455-63 15 Oct. 78.

55. WARSHAW J B. Insulin influences on fetal growth. Mead John son symp Perinat dev Med. (13): 40-4, 4-8, Jun 78. En: IM, 1979 p. 10. 156.

56. SCHULER K. When a pregnante women is diabetic. Antepartal care. Am J Nurs 79 (3) : 448-50, Mar. 79.

57. CASSAR J, et al. Simplified management of pregnancy complicated by diabetes. $\mathrm{Br} J$ Obstet Gynecol. 85 (8): 585-91, Aug. 78.

58. HOET J.J.et al. Clinical perspectives in the care of the pregnant diabetic patient. Ciba Found Symp. (63): 283-300, 30 Mar. 1 apr. 78.57 ref.

59. PEDERSON J, et al. Congenital malformations. The possible role of diabetes care outside pregnancy. Ciba Fund Symp (63): 265-71, 30 mar. 1 Apr. 78 En: IM, 1979 p. 10156

60. LOWY C. Diabetes in pregnancy (Letter): J.R. Soc Med 71 (7): 541-2, Jul. 78.

61. COUSTRAN D, et al. The diabetic pregnancy. Mead Johnson Symp perinatal Rev Med (13): 70-3, 4-8, Jun. 78 En IM, 1979 p. 10156.

62. GABBE SG, et al. Diabetes and pregnancy (Letter): Obstet Gynecol 52 (3): 381 2 Sep. 78.

63. ZARATE $A$, et al. Treatment of the pregnant diabetic patient Gac Med Mex 114 (4): 147-56, Apr. 78 (Español) 
64. BORBERG C, et al. Metabolic effects of beta sympathomimetic drugs and dexamethasone in normal and diabetic pregnancy. Br J Obstet Gynecol 85 (3): 1849, Mar. 78.

65. SMITH TC. Use of rotodrine in pregnant diabetics (Letter) $\mathrm{Br}$ Med J 2 (6089): 77017 Sep. 77.

66. CAPLAN RH, et al. Constant intravenous insulin infusion during labor and delivery in diabetes mellitus. Minn Med 60 (12): 861-3, Dec. 77 En: IM, 1978. p. 9802.

67. DRUNG MI. Management of diabetes in pregnancy (Letter): N Engl J Med 297 (16): 893-4, 20 Oct. 77.

68. COUSTAN DR, et al. Insulin therapy ofr gestational diabetes. Obstet Gynecol. 51 (3) 306-10, Mar. 78.

69. GABBE SG. Diabetes in pregnancy clinical controversies. Clin Obstet Gynecol 21 (2): 443-53, Jun. 78.

70. JOUGANATOS DM. et al. Diabetes in pregnancy metabolic changes and current management. J. Am Diet Assøc 73 (2): 168-71, Aug. 78 IM. 1978 p. 9802.

71. WATKINS P. J. Uiabetes in pregnancy a Synmposium diabetic control in pregnancy and labour. J R Soc Med 71 (3): 203-5, Mar. 78.

72. VINCE FP. Aspects of endocrinology. Practitioner 220 (1316): 279-83 Feb. 78. (30 ref.).

73. BOEHN FH. et al. Coordinated metabolic and obstetric management of diabetic pregnancy South Med J. 71 (1): 37-42. Jan. 78.

74. TCHOBROUTSKY C. Practical management during pregnancy of the insulin dependent diabetic. Acquis Med Recent. 79-84, 1977 (Francés): En: IM, 1978 p. 9802
75. ROVERSI, GD, et al. Diabetes in the mother and perinatal risk in pregnancy in diabetics by seeking strict metabolic equilibrium on the maternal disease 479 cases (1963): (1975): Rev Med Suisse Romande. 97 (8): 401-412, Aug. 77 (Francés). En IM. 1978 p. 9802.

76. GABBE, STEVEN G. Diabetes en el embarazo: conversaciones clínicas. Actualidad en Obstetricia y Ginecología. La Habana 3 (II): 21-34, Nov. 79

77. GOMEZ T. GUSTAVO y BRITTO, RODRIGO. Diabetes y embarazo. Rev. Col. Obstet Ginecol Bogotá 31 (2): 104-12, Mar-Abr. 80.

78. VALDES AMADOR, LEMAY A. y otros. La diabética subclínica embarazada. Su tratamiento, una incónigta (Comunicación preliminar): Revista Cubana de Obstetricia y Ginecología. La Habana 4 (I): 41-51, Ene-Mar.78.

79. MARQUEZ GUILLEN, ANTONIO Y otros. Nuestra experiencia con el tratamiento de la insulina prehipoglicemiante en el manejo de la gestante diabética. Comunicación preliminar. Revista Cubana de Obstetricia y Ginecología. La Habana 4 (2): 133-44, Abri.-Jun. 78.

80. VALDES AMADOR, LEMAY A. y otros. Movilización del colesterol y triglicéridos en la gestante diabética. Estudio de 86 pacientes con tratamiento prehipoglicemiante. Revista Cubana de Obstetricia y Ginecología. La Habana 5 (I): 1-12, Ene-Mar. 79.

81. WHO expert committee on diabetes mellitus - second report world health organization Geneva 1980.

82. BELITZKY R. et al. col. Normas perinatales de asistencia integral de laembarazada diabética, del parto y del hijo. Publicación científica CLAP No. 831. 
83. TEMESIO P., BELITZKY R. Diagnóstico y clasificación de la diabetes mellitus.

84. FESCINA R. Evaluación del crecimiento fetal en gestantes diabéticas por ecografía y clínica. Doc Int. CLAP 25/81.

85. ARISMENDI DE Q. I. TAVARES NORMA. Diabetes y embarazo aspectos perinatales. Publicación científica CLAP 8481979.

87. TEMESIO P. et col. Estudio de la $\mathrm{Hb}$ Al durante el embarazo de la diabética -CLAP 920-81 (1).

88. ANTORIA TEREZA. Nutrición y dieta en diabetes $y$ embarazo. CLAP. 920 81 (11).

89. BUSTOS RAUL. Evaluación de la maduración del pulmón fetal CLAP 920 81 (4).

86. LAWRENCE R. Maternal diabetes 1980. 